Tohoku J. exp. Med., 1965, 86, 238-246

\title{
Über chronische idiopathische interstitielle Myokarditis
}

\author{
Von \\ Takeshi Kurobane und Kazunori Nagai \\ Aus der Pathologischen Abteilung des Forschungsinstitutes für \\ Tuberkulose, Lepra und Krebs., Tohoku Universität, \\ Sendai; Direktor: Prof. T. Kurobane
}

(Received for publication, April 6, 1965)

\begin{abstract}
Ein seltener Sektionsfall von chronischer idiopathischer interstitieller Myokarditis wurde berichtet. Es handelt sich um einen 7 jährigen Junge, der über heftigen Bauchschmerz und Erbrechen klagte und in der Narkose vor einer Bauchoperation plötzlich ad exitum kam. Bei der Sektion fanden sich starke Dilatation und geringe Wandverdickung der beiden Herzkammern. Histologisch wurden im Myokard diffuse starke Vermehrung des Interstitiums sowie Ödem und Rundzelleninfiltration festgestellt. Daneben findet man vereinzelte fettige Degeneration der Herzmuskelzellen und fleckförmige Endokardverdickungen, die als sekundäre Prozesse anzusehen sind. Ausserdem wurden in diesem Fall Vergrösserung des Thymus und generalisierte Lymphknotenschwellung beobachtet. Auf Grund der histologischen Untersuchung nehmen wir an, dass die Veränderungen in Myokard, Thymus und Lymphknoten von gleicher infektioser Natur sind.
\end{abstract}

Seit Fiedler $(1900)^{1}$ über diffuse interstitielle Myokarditis von unklarer Ätiologie berichtet hat, finden sich in der Literatur zahlreiche Arbeiten über solchen Fälle. Dabei bezeichnet man diese Krankheit als Fiedlersche Myokarditis, primäre interstitielle Myokarditis, idiopathische Myokarditis, perniziöse Myokarditis, diffuse Sklerose des Myokardes usw. Die klinsiche Diagnose dieser Erkrankung gelang fast nie, so dass alle bisher mitgeteilte Fälle erst autoptisch und vor allem histologisch diagnostiziert worden sind. Nach allgemeiner Ansicht finden sich diese Myokarditisform meist beim Erwachsenen und manchmal auch beim Säuglinge. Dagegen wird sie bei älteren Kindern nur äusserst selten beobachtet. Wir möchten hier über unseren eigenen Fall von chronischer idiopathischer interstitieller Myokarditis bei einem sieben jährigen Jungen berichten, der gleichzeitig auch Schwellung von Thymus und Lymphknoten aufwies.

EIGENER FALL

Klinische Angaben

Es handelt sich um einen 7 Jahre und 9 Monate alten Knaben, der in der

黑羽 武，永节一微 
körperlichen Entwicklung etwas zurückblieb und oft eine vermehrte Ermüdbarkeit zeigte. Es bestand aber keine Zyanose. Familienanamnese: Grossvater mit 59 Lebensjahren an Herzkrankheit verstorben, Eltern angeblich gesund. Das Kind war bei der Röntgenreihenuntersuchung am 8. Juli 1963 wegen eines abnormen Herzschattens aufgefallen und wurde mit diesem Befund am 20. Juli in unsere Klinik aufgenommen. Keine besondere subjektive Symptome. Auskultatorisch hörte man aber ein lautes systolisches Geräusch mit Maximum im 4. ICR links parasternal. Röntgenologisch waren beide Vorhöfe und beide Kammern des Herzens beträchtlich vergrössert (Abb. 1). Deshalb wurde zunächst der Verdacht eines Vorhofseptumdefektes nahegelegt. Durch Herzkatheterismus konnte man aber feststellen, dass es kein Septumdefekt besteht. Die Blutsenkung betrug 4/14 mm nach Westergren. Am 18. August klagte der Patient über heftige Bauchschmerzen und zeigte häufige Erbrechen. Wegen Verdacht auf einen Tleus musste er sich am 20. August einer Laparotomie unterziehen. In der Narkose mit Äther starb er aber an plötzlich eingesetztem Herzversagen.

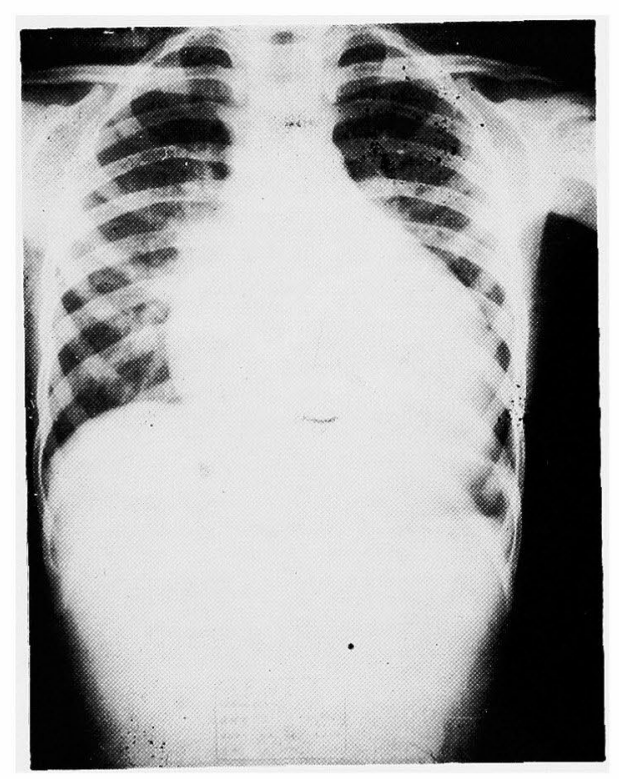

Abb. 1. Röntgenbild. Starke Vergrösserung des Herzschattens.

\section{Pathologisch-anatomische Diagnose:}

Chronische idiopathische diffuse interstitielle Myokarditis. Starke Vergrösserung des Thymus. Mässig starke, generalisierte Lymphknotenschwellung. Stauung der Lunge, Leber und Niere. Auszug aus dem Sektionsprotokoll: $111 \mathrm{~cm}$ grosse, $15 \mathrm{~kg}$ schwere Leiche eines 7 Jahre und 9 Monate alten Knaben. 
Herz: Epi- und Perikardüberzug glatt und spiegelnd. Im Epikard sieht man einige miliargrosse petechiale Blutungen. Herzbeutelflüssigkeit ist klar und leicht vermehrt. Das Herz ist ziemlich stark vergrössert $(11,5 \times 9,0 \times 4,5 \mathrm{~cm})$ und wiegt 200 g. Die Herzspitze ist von der linken Kammer gebildet und etwas abgerundet. Beide Herzventrikel sind stark dilatiert, links stärker als rechts. Die Herzmuskulatur ist von relativ weicher Konsistenz und blassbräunlicher Farbe. Sie hat einen eigenartigen Glanz. Ausserdem sieht man in der Muskulatur oft trübe, blassgraurote Bezirke. Die rechte Kammerwand hat eine Dicke von $0,5 \mathrm{~cm}$, die linke Kammerwand eine solche von 1,2 cm. Auch die Papillarmuskeln sind leicht verdickt. Am Endokard beider Herzventrikel finden sich manchmal fleckförmige, grauweisse Verdickungen. Die Klappen sind sämtlich zart und schlussfähig. Sehnenfäden nicht verkürzt. Das Foramen ovale ist geschlossen.

Das Thymus ist deutlich vergrössert $(6,5 \times 5,0 \times 3,0 \mathrm{~cm})$ und wiegt $40 \mathrm{~g}$. Die Schnittfläche des Thymus ist von grauer bis grauroter Farbe und zeigt einen deutlichen Läppchenbau.

Zahlreiche Lymphknoten in verschiedenen Körperregionen, besonders Mesenterial-, Lungenhilus- und Paratracheallymphknoten, sind mässig stark angeschwollen. Die Lymphknotenschwellung erreicht manchmal bis bohnengross, aber zeigt niemals eine Konfluierung. In der Schleimhaut des Ileums und des Colons finden sich zahlreiche angeschwollene Solitärfollikel. Lymphfollikel der Milz sind reichlich und deutlich zu sehen.

Das Peritoneum ist glatt und spiegelnd. Weder Verwachsung noch Ascites. Stenose oder Verschluss des Magendarm-Traktes ist nicht feststellbar. Sonst findet man in Lunge, Leber und Niere eine mässig starke Stauung.

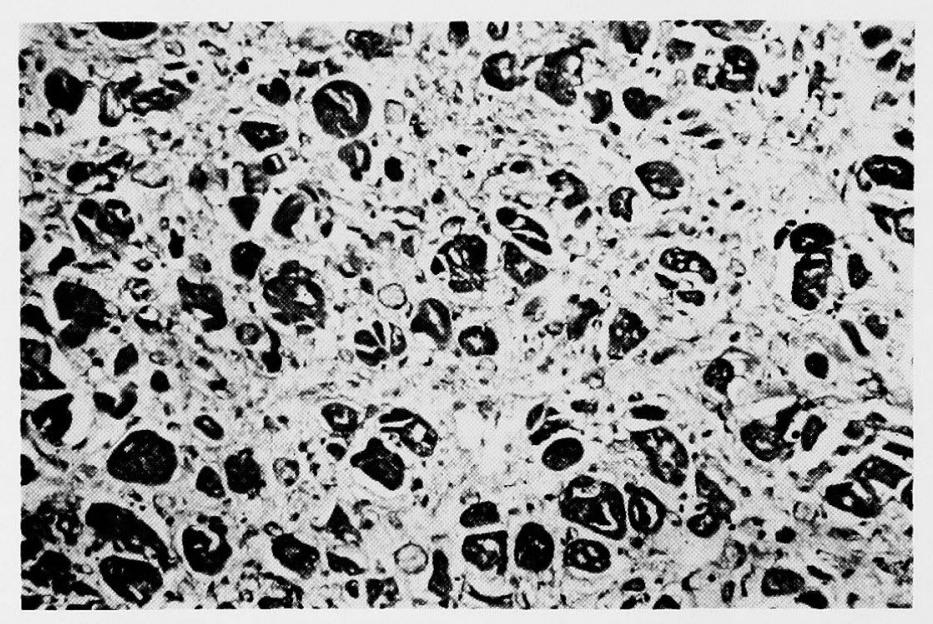

Abb. 2. Myokard. Starke diffuse Vermehrung des Interstitiums und geringe Rundzelleninfiltration. H.-E.-Färbung. $100 \times$. 


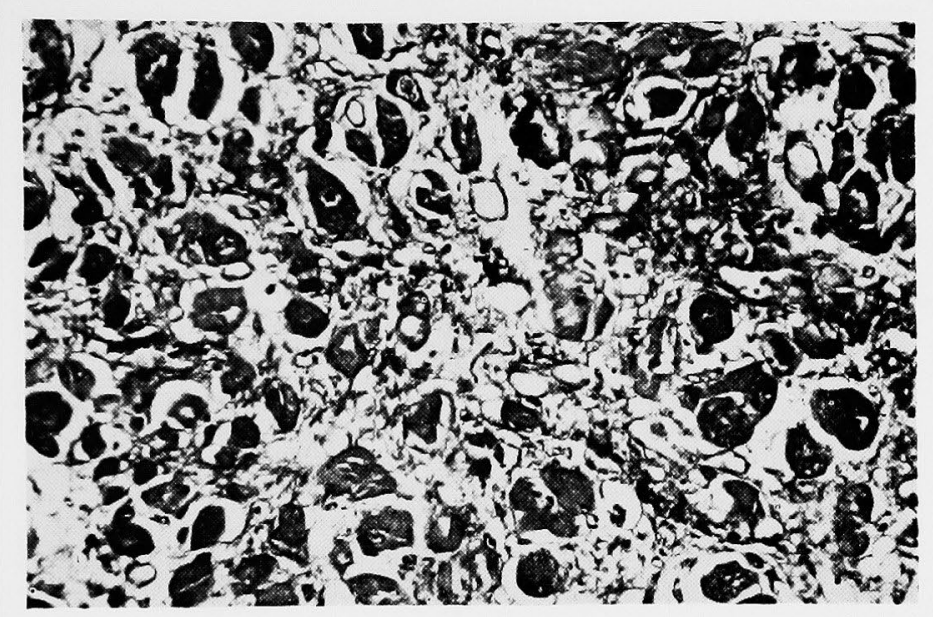

Abb. 3. Myokard. Kollagenfasern im Interstitium sind relativ locker angeordnet. Kleine und grössere Hohlräume zwischen den Fasern. van Gieson-Färbung. 100 .

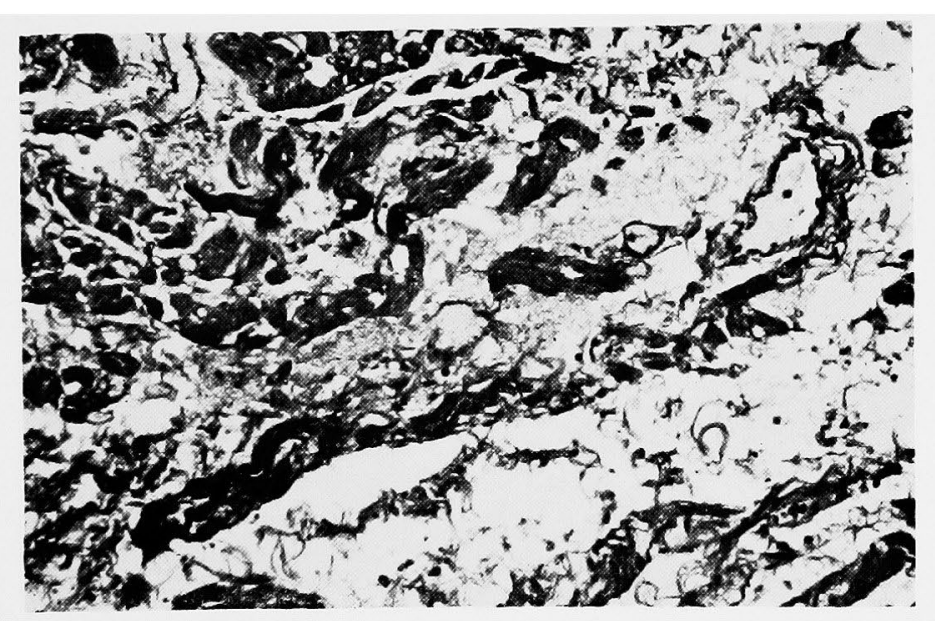

Abb. 4. Myokard. Starkes Ödem bzw. Auflockerung des Interstitiums. H.-E.-Färbung. 100 . .

\section{Histologischer Befund:}

Herz: Im Myokard finden sich starke diffuse Vermehrung des Interstitiums und geringe Lymphocyteninfiltration (Abb.2). Das Interstitium besteht vorwiegend aus Kollagenfasern und enthält relative wenig Gitterfasern. Elastische Fasern sind fast nicht nachweisbar. Im Vergleich mit der gewöhnlichen Narbe sind hier die Kollagenfasern feiner und locker angeordent (Abb. 3). Zwischen den Fasern wird häufig eine ziemlich grosse Lücken- bzw. Hohlraumbildung, die auf das Vorliegen 


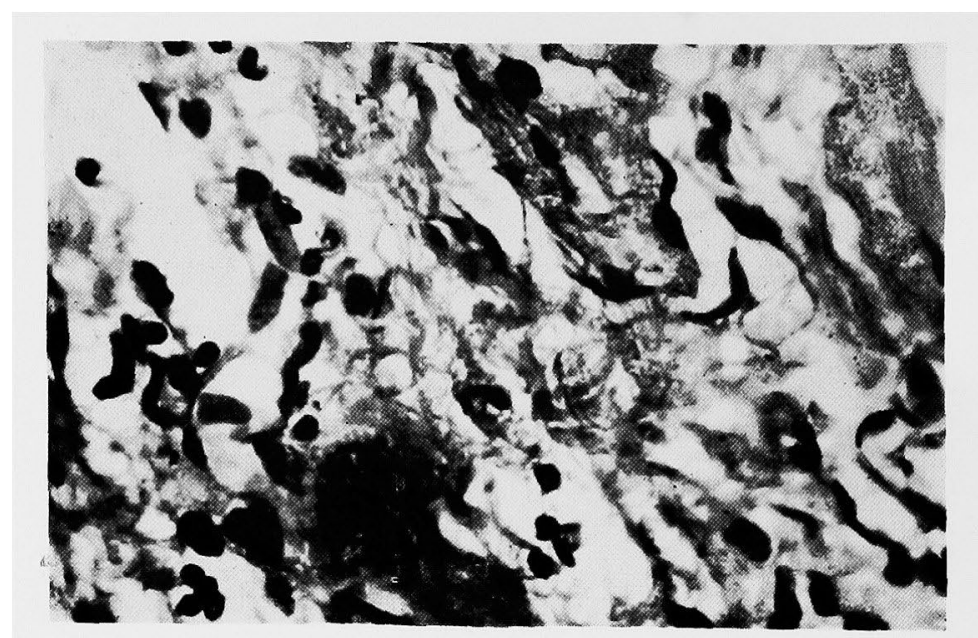

Abb. 5. Myokard. Muskelfasern sind aufgelockert. Links unten sieht man eine Muskelzelle mit dunkel gefärbtem riesenhaftem Kern. H.-E.-Färbung. 400 .

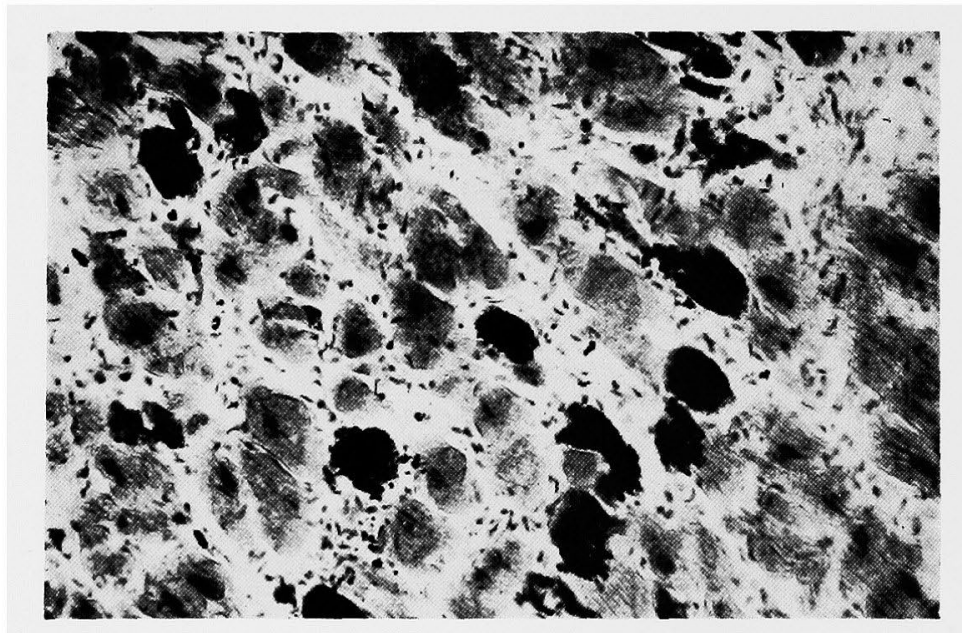

Abb. 6. Myokard. Vereinzelte fettige Degeneration der Muskelfasern. Sudan-Färbung. $100 \times$.

eines interstitiellen Ödems hinweist. Gelegentlich findet man auch ein deutliches Ödem des Interstitiums (Abb. 4). Blutgefässe und Kapillaren im Interstitium sind erweitert und mit Blut oder seröser Flüssigkeit angefüllt. Die Muskelfasern, die vom vermehrten Bindegewebe dicht umgeben sind, zeigen oft eine Atrophie oder im Gegenteil eine Hypertrophie. Nicht selten beobachtet man auch Muskelfasern mit dunkel gefärbtem riesengrossem Kern (Abb. 5). Bei der Sudan-Färbung kann an den Herzmuskelfasern nur ganz vereinzelte fettige Degeneration beobachtet wer- 


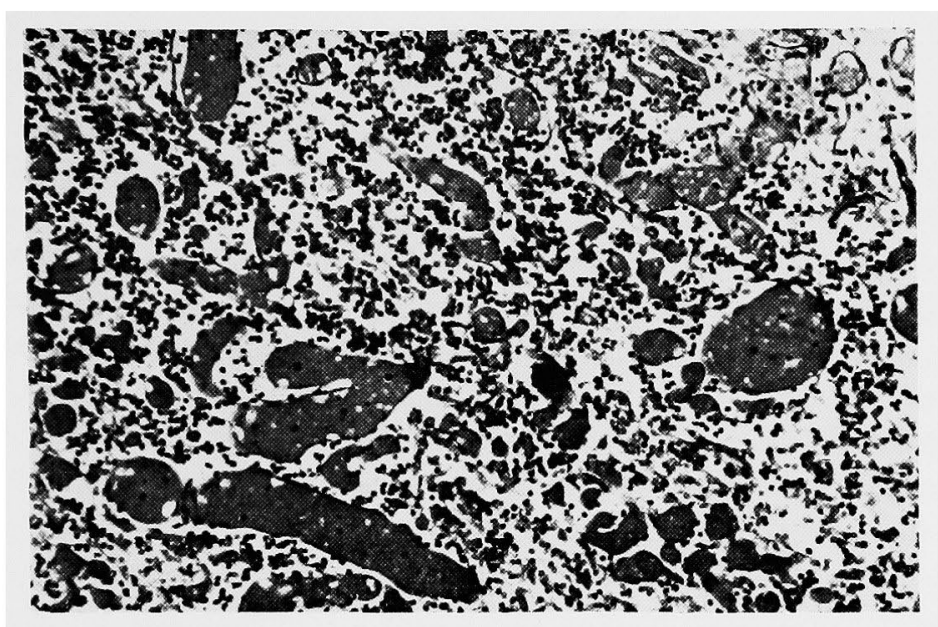

Abb. 7. Thymus. Hochgradige Hyperämie. H.-E.-Färbung. $100 \times$.

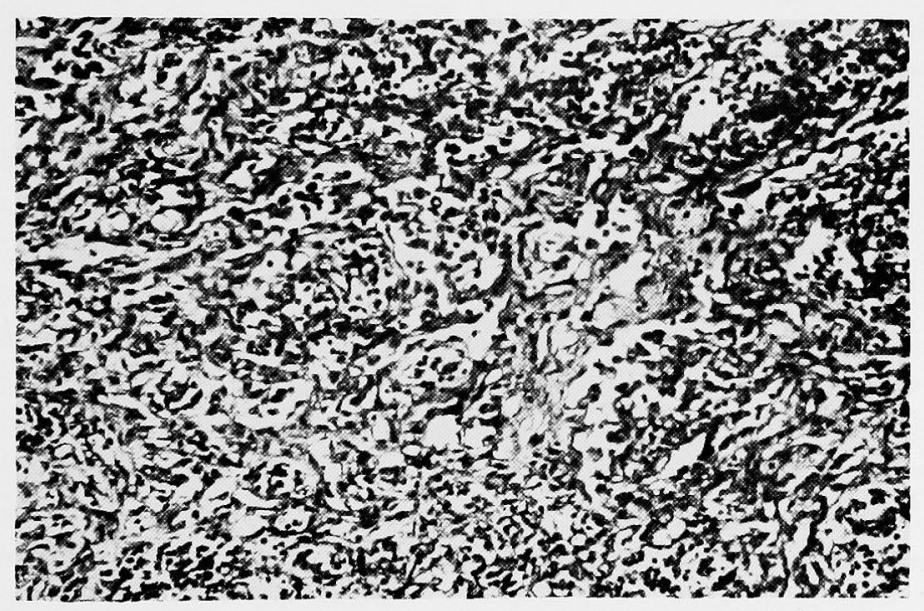

Abb. 8. Lymphknoten. Fibröse Veränderung der Pulpa.

H.-E.-Färbung. 100

den (Abb. 6). Solche Veränderungen sind in beiden Herzkammern nachweisbar, aber in der linken Kammer stärker ausgeprägt. Auch in der Wand beider Herzvorhöfe wurde der gleiche Befund erhoben. Was die Verteilung der Veränderungen innerhalb der Herzwandschicht anbetrifft, so kann man sagen, dass die äussere, perikardnahe Schicht im allgemeinen etwas stärker betroffen ist als die innere, endokardnahe Schicht. Im Epi- und Perikard findet man keine nennenswerte Veränderung, während das Endokard oft stellenweise eine mässig starke Verdickung zeight. Diese Endokardverdickung besteht aus elastischen und kollagenen 
Fasern und weist keine Zellinfiltration auf.

Das Thymus hat einen normalen Läppchenbau und zeigt eine starke Hyperämie (Abb. 7). Lymphocyten in der Rinde sind reichlich vorhanden, aber man findet hier keinen Sekundärfollikel. Im Mark liegen zahlreiche Hassallsche Körperchen.

Die Lymphknoten zeigen ebenfalls eine starke Hyperämie. Lymphocyten sind in der Pulpa relativ locker gelagert; Retikulummaschen der Pulpa sind teilweise erweitert. Man findet oft in Pulpa und Sinus eine deutliche Retikulumzellenvermehrung, die zum Teil zur Fibrose führt (Abb. 8). Der Lymphsinus, besonders der Marksinus, ist stark erweitert und zeigt eine Stauung von Lymphe.

In der Milz sind zahlreiche Lymphfollikel ohne helle Zentren zu sehen. Aber innerhalb der Follikel liegen die Iymphocyten nicht so dicht. In der roten Milzpulpa findet sich eine starke Hyperämie.

In der Submucosa des Darmes sind reichlich Lymphknötchen vorhanden, die ödematös angeschwollen sind.

Die Lungenalveolen sind verengert und enthalten oft grosse, mit Hämosiderin beladene Rundzellen. Die Alveolarsepten sind hyperämisch, leicht verdickt und von Lymphocyten geringmässig infiltriert. Im peribronchialen bzw. arteriellen Bindegewebe finden sich oft follikelähnliche Lymphocytenanhäufungen.

In Leber, Niere und anderen Organen fanden wir ausser einer Hyperämie keine besondere histologische Veränderung.

\section{DISKUSSION}

Nach Fiedler ist die Prognose der akuten interstitiellen Myokarditis infaust und endet die Krankheit meist am 5. bis 17. Tage letal. Als klinische Symptome hat er Tachycardie, Dyspnoe, Zyanose, Herzerweiterung usw. angegeben. Pathologisch-anatomisch findet man dabei die Entzündung ausschliesslich im Myokard, die übrigen Organe bleiben frei oder werden nur sekundär befallen. Ätiologisch steht diese Krankheit mit anderen Krankheiten, wie z.B. Rheumatismus, Diphterie, Scharlach, Arteriosklerose u.a., in keinem Zusammenhang. Diese erstmals von Fiedler beschriebene Myokarditis wurde neuerdings von zahlreichen Autoren, wie Saphir, ${ }^{2}$ Spahir, Wile und Reingold, ${ }^{3}$ House, ${ }^{4}$ Weber, Baldwin und Hall, ${ }^{5}$ v. Lutterotti ${ }^{6}$, Tedeschi und Stevenson, ${ }^{7}$ Williams, O'Reilly und Williams, ${ }^{8}$ Drenann, ${ }^{9}$ Karni, ${ }^{10}$ Gormsen, ${ }^{11}$ Faber und Fischer, ${ }^{12}$ Lichtenberger, ${ }^{13}$ Nikkilä und Pelkonen, ${ }^{14}$ eingehend untersucht. Auf der anderen Seite wurde von Fiebach, ${ }^{15}$ Boikan, ${ }^{16}$ Lindberg, ${ }^{17}$ Tannière und Bartman ${ }^{18}$ u.a.A. berichtet, dass es ausser solcher akuten Form der interstitiellen Myokarditis auch eine chronische Form gibt, die klinisch langsam verläuft und histologish hauptsächlich eine Fibrosierung des Myokardinterstitiums zeigt. Die letztere ist nach Fiebach und Boikan viel seltener als die akute Form. Die Unterscheidung zwischen akuter und chronischer Form ist nach Karni jedoch oft schwer, denn die Faservermehrung des Interstitiums 
ist nicht nur bei chronischer, sondern auch bei akuter Form meist nachweisbar. Die Ätiologie dieser Erkrankung ist heute noch unklar, aber man hat oft eine Virusinfektion angenommen (Gormsen, v. Lutterotti, Tannière und Bartman). Unser eigener Fall entspricht der chronische Form der Fiedlerschen Myokarditis: klinische Angaben sowie histologische Befunde sprechen dafür, dass der Herzprozess bei unserem Fall schon relativ lange bestanden hatte.

In Bezug auf die Häufigkeit gilt die idiopathische interstitielle Myokarditis als seltene Krankheit. Gore und Saphir ${ }^{19}$ fanden z.B. die Erkrankung nur in 43 von gesamten 1,402 Myokarditisfällen. In Japan wurden bis jetzt nur 3 Fälle mitgteilt (Kato, ${ }^{20}$ Arima ${ }^{21}$ und Otsu et al. ${ }^{22}$ ). Ferner hadelt es sich bei den meisten berichteten Fällen um Erwachsenen und Säuglinge. Lindberg, Weber, Saphir, v. Lutterotti, Tanniere u.a. haben darauf hingewiesen, dass diese Myokarditis nur ausnahmsweise bei älteren Kindern vorkommen kann. Daraus geht deutlich hervor, dass unser Fall, 7 jähriger Knabe mit chronischer idiopathischer interstitiellen Myokarditis, zu grossen Seltenheiten gehört.

Die histologischen Veränderungen bei dieser Erkrankung lokalisieren sich in der Hauptsache im Interstitium und die Herzmuskelfasern selbst weisen nach viele Autoren keine Degeneration auf. Dagegen fanden Lindberg, Saphir und Hafner $^{23}$ nicht so selten verschiedene Degenerationen an den Muskelfasern, obwohl sie geringmässig sind. Auch in unserem Falle konnten wir vereinzelt fettige Degeneration der Muskelfasern nachweisen. Mit allen anderen Autoren übereinstimmend fassen auch wir diese Herzmuskeldegeneration als sekundärer Prozess auf. Denn die Degeneration ist nur an solchen Musklfasern zu erkennen, die im vermehrten Bindegewebe fest eingesehlossen sind. Sie ist im Bereich mit weniger stark ausgeprägter Interstitiumvermehrung nicht nachweisbar. Epi- und Endokard sind meistens an dem Krankheitprozess nicht mitbeteiligt. Aber House, Lichtenberger sowie Tannière und Bartman haben in ihren Fällen von idiopathischer interstitieller Myokarditis Fibroelastose des Endokardes gefunden. Auch in unserem Falle haben wir herdweise Endokardverdickungen festgestellt, die aus elastischen und kollagenen Fasern bestehen. Wir nehmen mit Hadorn und Müller $^{24}$ sowie Sanders ${ }^{25}$ an, dass solche Herde sekundär durch Übergreifen der Muskelschichtveränderung auf das Endokard entstanden.

Riesenfeld, ${ }^{26}$ Cellen, ${ }^{27}$ Fahr $^{28,29}$ und Rieder ${ }^{30}$ fanden im Myokard der plötzlich gestorbenen Fällen von Status thymico-lymphaticus bedeutende lymphocytäre Infiltrationen, die als Todesursache ihrer Fälle angesehen werden können. Riesenfeld und Ceelen glauben, dass solche Myokardveränderung mit dem Status thymico-lymphaticus in enger Beziehung steht. Nach diesen Autoren soll die Lymphocyteninfiltration im Myokard bei Status thymico-lymphaticus Teilerseheinung oder toxische Vorgänge der allgemeinen lymphatischen Hyperplasie darstellen. Dieser Meinung hat sich neulich auch Kato angeschlossen. Dagegen betonen Fahr und Rieder, dass es sich nicht um eine lymphatische Hyperplasie, sondern um eine 
Entzündung des Myokardes handelt. Ferner lehnen Vischer, ${ }^{31}$ Saphir und Arima die ursächliche Rolle des Status thymico-lymphaticus für die Entstehung der interstitiellen Myokarditis ab. In unserem Fall fanden wir auch beträchtliche Vergrösserungen von Thymus, Lymphknoten und anderen Lymphapparaten. Makroskopisch entspricht deshalb das Bild dem Status thymico-lymphaticus. Histologisch sind aber Hyperplasie der Lymphfollikel bzw. deutliche Keimzentren nicht nachweisbar. Die Lymphocyten sind im lymphatischen Gewebe locker angeordnet. Demgegenüber findet man in diesen Organen Hyperämie, Ödem und Faservermehrung, die der Interstitiumveränderung des Myokardes etwa entsprechen. Es ist daher anzunehmen, dass bei unserem Fall die Veränderungen in Myokard, Thymus und Lymphknoten durch ein und dieselbe Infektion hervorgerufen wurden.

\section{Literaturverzeichnis}

1) Fiedler, A. Zbl. inn. Med., 1900, 21, 212.

2) Saphir, O. Arch. Path., 1941, 32, 1000.

3) Saphir, O., Wile, S.A. \& Reingold, I.M. Amer. J. Dis. Child., 1944, 67, 294.

4) House, R.K. Amer. J. Path., 1948, 24, 1235.

5) Weber, M.W., Baldwin, J.S. \& Hall, J.W. Pediatrics, 1949, 3, 829.

6) v. Lutterotti, M.Z. Kreisl. Forsch., 1950, 39, 417.

7) Tedeschi, C.G. \& Stevenson, T.D. New Engl. J. Med., 1951, 244, 352.

8) Williams, H., O'Reilly, R.N. \& Williams, A. Arch. Dis. Child., 1953, $28,271$.

9) Drennan, J.M. ibid., 1953, 28, 288.

10) Karni, H. Acta med scand, 1954, 149, 243.

11) Gormsen, H. Acta path. scand., 1955, Suppl. 105, 30.

12) Faber, V. \& Fischer, S. Acta med scand., 1956, 154, 135.

13) Lichtenberger, E. J. Mt. Sinai Hosp., 1957, 24, 1001.

14) Nikkilä, E.A. \& Pelkonen, R. Acta med. scand., 1959, 165, 421.

15) Fiebach, R. Virchow's Arch., 1921, 233, 57.

16) Boikan, W.S., ibid., 1931, 282, 46.

17) Lindberg, K. Acta med. scand., 1938, 95, 281.

18) Tannière, M. Th. \& Bartman, J. Acta paediat. Belg., 1959, 13, 329.

19) Gore, I. \& Saphir, O. Amer. Heart J., 1947, 34, 827.

20) Kato, A. Tr. Soc. Path. Jap. (Jap.), 1955, 44, 482.

21) Arima, Y. Yokohama Igaku (Jap.), 1960, 11, 58.

22) Otsu, S., Shozawa, T., Seki, M., Takeda, Y. \& Murata, K. Nippon Junkankigakkai Zasshi (Jap.), 1960, 24, 670.

23) Hafner, A. Dtsch. Arch. klin. Med., 1922, 138, 236.

24) Hadorn, W. \& Müller, P. Arch. Kreisl.forsch., 1961, 35, 186.

25) Sanders, V. Arch. intern. Med, 1963, 112, 661.

26) Riesenfeld, A. Jb. Kinderheilk, 1917, 86, 419.

27) Ceelen, W. Berl. klin. Wschr., 1920, 57, 197.

28) Fahr, Th. \& Kuhle, J. Virchow's Arch. 1921, 233, 286.

29) Fahr, Th. Zbl. Path., 1921/1922, 32, 505.

30) Rieder, H. $J b$. Kinderheilk., 1922, 97, 9.

31) Vischer, M. Zbl. Path., 1924/1925, 35, 67. 